OPEN ACCESS

Edited by:

James W. Grau,

Texas A\&M University, United States

Reviewed by:

Vince D. Calhoun

University of New Mexico,

United States

Afra Wohlschlaeger,

Technische Universität München,

Germany

*Correspondence:

Oleksandr V. Popovych

o.popovych@fz-juelich.de

Received: 27 June 2018 Accepted: 17 December 2018

Published: 10 January 2019

Citation

Popovych OV, Manos T, Hoffstaedter F and Eickhoff SB (2019) What Can Computational Models Contribute to Neuroimaging Data Analytics?

Front. Syst. Neurosci. 12:68 doi: $10.3389 /$ fnsys.2018.00068

\section{What Can Computational Models Contribute to Neuroimaging Data Analytics?}

\author{
Oleksandr V. Popovych ${ }^{1,2 *}$, Thanos Manos ${ }^{1,2}$, Felix Hoffstaedter ${ }^{1,2}$ and Simon B. Eickhoff ${ }^{1,2}$ \\ ${ }^{1}$ Institute of Neuroscience and Medicine - Brain \& Behaviour (INM-7), Research Centre Jülich, Jülich, Germany, ${ }^{2}$ Institute of \\ Systems Neuroscience, Medical Faculty, Heinrich-Heine University Düsseldorf, Düsseldorf, Germany
}

Over the past years, nonlinear dynamical models have significantly contributed to the general understanding of brain activity as well as brain disorders. Appropriately validated and optimized mathematical models can be used to mechanistically explain properties of brain structure and neuronal dynamics observed from neuroimaging data. A thorough exploration of the model parameter space and hypothesis testing with the methods of nonlinear dynamical systems and statistical physics can assist in classification and prediction of brain states. On the one hand, such a detailed investigation and systematic parameter variation are hardly feasible in experiments and data analysis. On the other hand, the model-based approach can establish a link between empirically discovered phenomena and more abstract concepts of attractors, multistability, bifurcations, synchronization, noise-induced dynamics, etc. Such a mathematical description allows to compare and differentiate brain structure and dynamics in health and disease, such that model parameters and dynamical regimes may serve as additional biomarkers of brain states and behavioral modes. In this perspective paper we first provide very brief overview of the recent progress and some open problems in neuroimaging data analytics with emphasis on the resting state brain activity. We then focus on a few recent contributions of mathematical modeling to our understanding of the brain dynamics and model-based approaches in medicine. Finally, we discuss the question stated in the title. We conclude that incorporating computational models in neuroimaging data analytics as well as in translational medicine could significantly contribute to the progress in these fields.

Keywords: neuroimaging, resting state, mathematical models, brain dynamics, functional connectivity, simulation, high-performance computing

\section{DATA-DRIVEN APPROACH}

Advances in neuroimaging techniques and data analytics methods have led to a rapid advancement in the description of structural and functional properties of brain networks. The anatomical architecture of brain networks, i.e., their structural connectivity (SC), can be inferred from diffusion weighted magnetic resonance imaging (dwMRI). In particular, fiber tracts reconstructed through tractography algorithms can serve as proxies for physical connections between brain regions at the meso- and macroscopic level (though see Jones et al., 2013; Thomas et al., 2014; Maier-Hein et al., 2017 for a discussion on the limitations of fiber reconstruction from dwMRI). Such 
structural information thus contributes a description of the underlying physical organization of the brain on top of which the time dynamics of neuronal activity can emerge. The latter can be measured by indirect, blood flow-based methods such as functional MRI (fMRI) and positron emission tomography (PET) as well as more direct recording of neuronal activity through electroencephalography (EEG), magnetoencephalography (MEG) or as local field potential (LFP) measured by implanted electrodes. While these methods differ substantially with respect to their spatial and temporal resolution (cf. Horwitz et al., 2000; Bandettini, 2009), they can all be used in two fundamental contexts in task-related and task-free examinations. Classically, subjects perform an active sensory, cognitive or motor paradigm while brain activity is recorded. This allows to probe context-dependent modulations of regional activity and network interactions, reflecting the effects of the respective tasks on functional and effective connectivity (Bressler and Menon, 2010; Park and Friston, 2013).

While massively growing our knowledge on functional specialization and integration over more than two decades, taskbased imaging also suffers from several drawbacks. Brain activity in a task setting is bound to the particular experimental context and hence limited in generalizability, re-usability as well as in practicality for imaging large cohorts or a clinical setting. Consequently, much focus has been devoted more recently to the acquisition and analysis of what has been termed the "taskfree" or "resting-state" activity of the brain. Making use of the constantly ongoing mental processes and the fact that brain networks hence coordinate their activity also in the absence of an explicit behavioral task, resting-state imaging (Biswal et al., 1995) has yielded new perspectives in the study of large cohorts and patient populations as well as establishing data-sharing in the field of neuroimaging. Consequently, large databases collected thousands of brain imaging records and behavioral information (Van Essen et al., 2013; Caspers et al., 2014). In the most common approach, i.e., resting-state fMRI, the temporal fluctuations of the blood oxygen level-dependent (BOLD) signals are related to each other by clear and robust correlation (e.g., by using Pearson correlation coefficient) across what are assumed to be functionally related brain regions coordinating their activity during sensory, motor or cognitive brain functions, i.e., functional connectivity (FC) between them (Biswal et al., 1995; Park and Friston, 2013; Varikuti et al., 2017).

Resting-state FC analysis has without doubt revolutionized the field of human brain imaging and has led to the presence of many new insights into inter-individual variability and the pathophysiology of brain disorders. For example, differences in the group-average resting-state FC patterns have been shown for disorders such as Parkinson's and Alzheimer's disease, stroke, depression and schizophrenia (Sorg et al., 2007; Konrad and Eickhoff, 2010; Tahmasian et al., 2015). Moreover, it has been demonstrated, that individual patterns of BOLD correlations in the absence of a structured task allow for the individual classification of previously unseen subjects as patients or healthy controls with good accuracy (Arbabshirani et al., 2017; Pläschke et al., 2017). Together with the fact that resting-state measurements are attainable even in comparably ill patients, this opens an avenue for a clinical application toward objective diagnosis and may even allow to provide prognoses of disease development on an individual basis (Woo et al., 2017; Yahata et al., 2017). Finally, investigating FC patterns of spontaneous brain activity at the single-subject level, individualized targets for interventional approaches using invasive or non-invasive neuromodulations may provide a road to more individualized, neurobiologically informed treatments (Fox et al., 2014).

In healthy subjects, resting-state FC can be used to build data-driven connectome-based predictive models to differentiate between subjects and to predict individual behavior from the brain activity (Shen et al., 2017). Robust and reliable individual variability of the FC profiles at "rest" permits a relatively accurate identification of individual subjects from a large group (Finn et al., 2015). The contemporary research in this framework is focused on prediction of fluid intelligence of individual subjects, performance in specific tasks, personality traits and brain aging from resting-state fMRI data (Dosenbach et al., 2010; Finn et al., 2015; Dubois and Adolphs, 2016; Tavor et al., 2016; Liem et al., 2017). This also has an important clinical relevance, for instance, in distinguishing between normal aging and disease.

In spite of its undisputed success, however, resting-state fMRI is also a field marked by substantial controversy over the appropriate analyses methods and the interpretation of observed patterns. For the task-based imaging there could exist a relatively clear time frame and related expectations, where and what is going to happen in the brain and its relation to behavioral states across subjects. For the resting-state measurements, the absence of an external reference by experimentally defined time-points and behavioral states together with enhanced inter-individual variability renders the whole field devoid of any gold-standard or ground-truth. This makes the resting-state data processing and analysis and interpretation of results even more challenging than for the task-based paradigm.

It may hence not surprise that many of the most cited neuroimaging papers over the last years pertain to the characterization of (potential) artifacts, evaluation of processing or analysis methods and inferences that can be drawn from these (Murphy et al., 2013; Satterthwaite et al., 2013; Power et al., 2014; Salimi-Khorshidi et al., 2014). For example, it is generally agreed that artificial sources of variance caused by subject movement as well as by various sources of physiological noise (such as heartbeat, breathing) may strongly influence the observed BOLD correlations and need to be accounted for. How this goal is best achieved, though, is a matter of conjecture as there is no gold standard given that we don't know how the BOLD signal would look like without these influences. Consequently, methods for characterizing and removing what should be considered structured noise within resting-state time series have, e.g., proxied the effects of movement for example by differences between high- and low-moving subjects (Satterthwaite et al., 2013). There is an ongoing discussion on how to deal with global signal changes, as it can be shown that when these are not accounted for, virtually all brain regions exhibit functional correlations with each other while removal of global signals leads to pronounced anti-correlations (Varikuti et al., 2017). The latter are questioned as spurious by some 
(Murphy et al., 2009) and highlighted as antagonistic large-scale systems by others (Fox et al., 2009). Finally, in particular in the context of predicting individual phenotypes and ultimately precision medicine, one of the key prerequisites is to reduce the dimensionality from all possible interactions among $>$ 200.000 voxels onto a tractable number of region-by-region connections. This is usually done through referencing to a brain atlas providing the respective parcellation, but unfortunately, there is no general consensus as to which parcellation would be most appropriate, see the papers (Thirion et al., 2014; Eickhoff et al., 2018a,b) for review and references therein. In fact, both the approach for atlas generation with respect to imaging modalities and parcellation techniques (restingstate, structure, geometry, independent/principal components analysis (ICA/PCA), clustering etc.) and the ensuing granularity vary considerably across studies performing connectome-based resting-state analyses.

In summary, while the potential of resting-state fMRI to contribute to our understanding of the normal and diseased brain is extremely high, many questions on how to attain this goal are still unresolved.

\section{MODEL-BASED APPROACH}

The achievements of the data-driven approach discussed above can significantly be extended if mathematical models get involved in the analysis to gain possible mechanistic explanations of the dynamics and properties of the brain resting-state networks (RSN). A thorough exploration of possible dynamical repertoires that the models can support and their relation to the phenomena observed in the data can help to formulate predictions and hypotheses that can serve as a basis for further experimental investigation and data analysis. A number of models of different complexity have been suggested for RSN neural population dynamics including phase oscillators (Cabral et al., 2011; PonceAlvarez et al., 2015), limit-cycle oscillators (Bettinardi et al., 2017; Deco et al., 2017), mean-field and spiking neuron models taking into account contributions of excitatory and inhibitory neurons located within the network nodes (Deco and Jirsa, 2012; Deco et al., 2014b; Glomb et al., 2017), and others (Deco et al., 2008; Sanz-Leon et al., 2015). The individual nodes can be coupled to brain networks, where the inter-node connections can be derived from empirical data, which was coined as the brain structural connectome (Sporns et al., 2005). Such SC obtained from diffusion weighted imaging and tract tracing studies can be used to build a graph of the brain network defining an underlying topological structure of the model network (Knock et al., 2009; Bullmore and Bassett, 2011; Park and Friston, 2013).

The model parameters can be calibrated in such a way that the model dynamics closely replicates that of the brain networks extracted from the empirical data. Realistic power spectra and fluctuations of BOLD signal were obtained from the models simulating underlying electrical activity of the neuronal populations (Ghosh et al., 2008; Deco et al., 2009; Deco and Jirsa, 2012; Schirner et al., 2018), where an advance modeling can also be used to infer the neurophysiological mechanisms underlying neuroimaging signals (Schirner et al., 2018). At this, parameters of noise intensity, time delay in coupling, global coupling strength, and excitation-inhibition balance may play a crucial role. It was found that there can exist optimal parameter settings, where the matrix of empirical FC strongly correlates with that calculated from the data generated by the model (Deco and Jirsa, 2012; Deco et al., 2013; Nakagawa et al., 2013). The maximal correspondence between model and empirical data can emerge when the model is close to a critical point at which the system may undergo a bifurcation, a sudden qualitative change of its dynamics and engage in a multistable regime that is highly sensitive to perturbations (Deco et al., 2013; Golos et al., 2015; Cocchi et al., 2017). Noise-driven exploration of the state space in the vicinity of attractors allows the emergence of dynamics observed in RSN activity (Ghosh et al., 2008; Deco et al., 2012, 2013; Golos et al., 2015).

FC patterns may strongly vary in time as we discuss below, and the static description of FC matrix obtained over long epochs $(\geq 5 \mathrm{~min})$ is distinguished by so-called dynamic FC reflecting time variations of correlations and characterized by the ongoing switching of network patterns (Hutchison et al., 2013; Allen et al., 2014; Cabral et al., 2017b). Analysis of dynamic FC for patients with schizophrenia and bipolar disorder has revealed different switching regimes as compared to healthy controls (Damaraju et al., 2014; Rashid et al., 2014), which cannot be obtained by the static connectivity analysis. The extraction, analysis, interpretation and application of dynamic FC are in the focus of investigation nowadays, see the recent comprehensive review (Preti et al., 2017).

In the framework of model-based approach, several recent studies (Hansen et al., 2015; Deco and Kringelbach, 2016; Deco et al., 2017; Jobst et al., 2017) modeled dynamic FC and showed that non-stationary connectivity dynamics can demonstrate a rich spatio-temporal structure and fast switching between a few discrete states as observed in empirical data. These studies also reported that the brain at rest operates at a maximal level of metastability (Deco and Kringelbach, 2016; Deco et al., 2017; Jobst et al., 2017), which can be referred to as an outof-equilibrium state characterized by a pronounced variability of phase configurations of the system and switching between different regimes of collective dynamics. It was suggested to be measured by the standard deviation of the Kuramoto order parameter reflecting the extent of synchronization in the networks across time (Deco and Kringelbach, 2016; Deco et al., 2017; Jobst et al., 2017). The concept of maximal metastability for the RSNs in the first place implies a state of maximum network switching and enhanced variability of synchronization in restingstate brain activity. Metastability is a fundamental property of transient brain dynamics and cognitive processes such as sequential decision making which can be modeled by a stable heteroclinic cycle involving complex metastable brain states (Rabinovich et al., 2008; Tognoli and Kelso, 2014). Therefore, fitting the extent of metastability and the properties of dynamic FC of the model to empirical data can provide an additional tool for validation and optimization of whole-brain models (Deco and Kringelbach, 2016; Deco et al., 2017; Jobst et al., 2017). 
The model-based approach can effectively be used for addressing neuropsychiatric disorders aiming to describe disease mechanisms (Deco and Kringelbach, 2014; Stephan et al., 2015). One example is schizophrenia which can be viewed as a brain network disorder (Friston and Frith, 1995; Calhoun et al., 2009; Lynall et al., 2010; van den Heuvel and Fornito, 2014). Simulations of whole-brain computational models demonstrated that clinical symptoms and changes in FC patterns observed in empirical data can be accounted for by a variation (decrease) of the global coupling strength in the model (Cabral et al., 2012b, 2013). On the other hand, the average power and variance of the global brain signal may increase in schizophrenia, which was replicated by dynamics of a computational model when local or global coupling strength increases (Yang et al., 2014; Anticevic et al., 2015). Modeling approaches can also be used to illustrate how the changes in SC due to long-term deep brain stimulation (DBS) in patients with Parkinson's disease (PD) can be reflected in resting-state brain dynamics and FC (van Hartevelt et al., 2014). Whole-brain computational models can help to reveal acute effects of DBS on resting-state brain dynamics when comparing DBS-On and DBS-Off regimes (Saenger et al., 2017). When comparing model parameters of local and global coupling as well as conduction velocity fitted to the functional data of stroke patients and healthy controls, a significant difference between these two parameter sets can be observed (Falcon et al., 2016). Furthermore, the coupling parameters correlated with long-term motor gains during recovery after stroke, which indicates that the model parameters can serve as biomarkers with potential predictive power for recovery after stroke (Falcon et al., 2016).

The potential of brain network modeling for diagnose, prognosis and treatment of neurological and psychiatric diseases is probably the most important perspective of this approach. The growing field of precision medicine is directed toward disease prevention and more predictive and personalized medicine as opposed to the treatment of patients mostly based on their segregation with respect to disease type or subtype (Duffy, 2016). Such personalized approach to the medical treatment would in particular require an involvement of personalized predictive computational models of individual patients. In this framework the concept of the virtual epileptic patient has recently been proposed (Jirsa et al., 2017; Proix et al., 2017), where the development of a personalized mathematical model for an epileptic patient was illustrated step by step. Such a model is based on the personalized SC and other indicators (e.g., lesions, epileptogenic zones, etc) and can be validated and optimized to reflect, explain and predict epileptic seizure propagation (Proix et al., 2017). Systematic explorations of the model parameter space can give an insight into how the clinically relevant parameters, such as neuronal excitability and coupling strength, might influence the number of seizures, their localization and propagation. Although the relation between the changes of the model parameters and impacts of the clinical intervention is not always evident, the models can nevertheless serve as a test bed for clinical hypothesis testing and thus provide the clinicians with an additional tool for hypothesis building and decision-making at brain intervention strategies. The knowledge obtained from the model-based investigation and data fitting, for example, for localization and extent of the epileptogenic zone can be combined with a data-driven approach and the experience of clinicians. This can contribute to the improvement of diagnosis and surgery outcome as well as to development of novel therapies (Jirsa et al., 2017; Proix et al., 2017).

Applying the model-based approach, brain stimulation techniques have been developed for counteracting abnormal neuronal synchronization characteristic for some neurological disorders including PD and tinnitus by desynchronization (Tass, 2003; Tass and Majtanik, 2006; Popovych and Tass, 2012; Ebert et al., 2014; Manos et al., 2018a,b). Desynchronizing stimulation techniques have been designed, tested and optimized with the help of mathematical models of neuronal populations. Parameters of stimulation intensity, timing and shape of the stimulation signal have systematically been varied and evaluated, which is hardly feasible in experimental and clinical setting. The hypotheses and predictions drawn from the modeling studies have successfully been tested in preclinical and clinical studies (Tass et al., 2012a,b; Adamchic et al., 2014) for different stimulation modalities including electrical DBS for PD and noninvasive acoustic stimulation for tinnitus.

A recent shift toward a closed-loop stimulation paradigm for DBS (Little et al., 2013; Rosa et al., 2015) has led to a more efficient counteracting of disease symptoms. The same clinical effects can be achieved for a significant reduction of the amount of the delivered stimulation current if the stimulation is administered when necessary in a demand-controlled way. This was preceded by theoretical development and modeling of closed-loop stimulation methods (Rosenblum and Pikovsky, 2004; Popovych et al., 2005, 2006). Model-based approach can further be used to optimize and adapt the stimulation techniques for clinical application, where they can be tested for realistic DBS signals (Popovych et al., 2017a,b; Popovych and Tass, 2018). Optimal parameter values can be found based on a thorough scan of parameter space of the models.

As for the research driven by the data, the model-based approach is not limited to the investigations of diseased states. Large-scale whole-brain computational models can provide an insight into how resting-state brain activity and FC patterns may evolve in aging (Nakagawa et al., 2013; Naik et al., 2017). Dynamics of the model and its parameters, for example, the coupling strength and the delay in coupling demonstrate consistent differences when comparing groups of young and old adults with high and low performance in cognitive tasks (Naik et al., 2017), which can be considered as potential model-based biomarkers for healthy aging.

In summary, mathematical dynamical models can be built based on brain structure and activity derived from the empirical data and can potentially be applied to any topic addressed by data-driven analysis. The derived models can however provide additional information and even explanations of the observed phenomena, which can contribute to our understanding of brain dynamics and treatment of diseases. 


\section{ENHANCING DATA ANALYTICS BY MODELS}

To build, validate and investigate models of brain networks, a number of computational tools have been developed including NEURON, NEST, BRIAN, and The Virtual Brain (TVB) (Carnevale and Hines, 2006; Gewaltig and Diesmann, 2007; Goodman and Brette, 2009; Sanz Leon et al., 2013), which combine biological relevance with up-to-date computational algorithms for high-performance computational clusters. For example, TVB is an open platform, where large-scale brain network models can be derived from the raw multi-modal imaging data, and an optimal match between empirical and simulated data, e.g., (dynamic) FC of RSNs, can be reached by an appropriate parameter calibration (Ritter et al., 2013; Sanz Leon et al., 2013; Sanz-Leon et al., 2015; Falcon et al., 2016).

The discussed dynamical models of brain networks are built on empirical SC establishing in such a way a natural link between brain structure and function. For sufficiently long scanning sessions, patterns of static FC of the spontaneous brain dynamics correlate with patterns of SC (Hagmann et al., 2008; Honey et al., 2009; Marrelec et al., 2016), and some properties of them can be inferred from each other (Hermundstad et al., 2013). This is also feasible for dynamic FC (Marrelec et al., 2016; Cabral et al., 2017b). However, the largely invariant SC can support many different $\mathrm{FC}$ patterns making the interdependence between them rather complicated (Park and Friston, 2013; Marrelec et al., 2016; Cabral et al., 2017a). For example, FC patterns most frequently observed in healthy older adults with good cognitive performance are less correlated with SC as compared to the connectivity patterns of poorly performing subjects (Cabral et al., 2017b).

Models can help to understand the correspondence between structural and functional connectivity (Honey et al., 2009). Homogeneous coupling strength and delay in coupling may lead to a strong structure-function correlation, whereas distributed delays and coupling strength between network nodes may cause a discrepancy between the observed activity patterns and underlying connectivity (Popovych et al., 2013; Ton et al., 2014). Therefore, one conclusion derived from theses modeling studies is that SC used for data analytics and mathematical modeling should be as precise as possible, especially, with regard to strength, delay and directionality of connections. This was also addressed by comparing large-scale brain simulations with models built on weighted undirected and directed structural connections (Knock et al., 2009), where the simulation in the latter case demonstrated more biologically realistic results.

Since investigation of the models is not restricted by the measured data, model parameters can be varied in a broad range to mimic possible scenarios of the evolution of brain states. For example, by modeling the hypothesis of structural disconnection in schizophrenia (Friston and Frith, 1995; Calhoun et al., 2009; Lynall et al., 2010; van den Heuvel and Fornito, 2014), parameters of the computational model can be varied from the states of strong to weak coupling or even by completely removing anatomical connections in the model (Cabral et al., 2012a). Such a computational experiment revealed changes in FC patterns also observed in empirical data (Lynall et al., 2010) and led to conclusion that disconnection-related neuropathologies can induce qualitatively similar changes in resting-state brain activity (Cabral et al., 2012a). From the opposite perspective, the modeling approach can be used to optimize SC configurations to obtain the best fit with FC from measured data by adding a few additional links and rewiring existing connections (Deco et al., 2014a). Such an approach may strongly contribute to the determination of empirical SC by predicting and suggesting possible anatomical links and their characteristics that are difficult to measure or to estimate from empirical data.

The above examples illustrate how the modeling can contribute to a mechanistic explanation of the structural and functional properties of the brain and their interrelation. With such an approach the emergent brain dynamics including resting state can be explained in terms of a few generic bifurcations (Cocchi et al., 2017) and assigned to a particular range of parameters that may vary depending on the brain state (Deco et al., 2013, 2017; Jobst et al., 2017). These parameters may have a well-founded relation to the brain characteristics such as global and local coupling, communication delay, noise intensity, heterogeneity of individual node dynamics and its excitationinhibition balance as well as external input or stimulation (Ghosh et al., 2008; Deco et al., 2009, 2014b; Popovych and Tass, 2012). It is thus very important to perform a detailed investigation of the derived models, which could be assisted by the computational tools mentioned above. Several attempts in this direction have explored the emergence of multistability in the large-scale brain models (Deco and Jirsa, 2012; Golos et al., 2015; Hansen et al., 2015). The concepts of multi- and meta-stability, which distinguish themselves by the coexistence of either attractors or saddles, respectively (Cocchi et al., 2017), are the main model-based hypotheses of the emergence of the resting-state fluctuations of the BOLD signals and FC matrices (Deco et al., 2012, 2017; Deco and Kringelbach, 2016).

Furthermore, models can help to address the question on how exactly the brain parcellation (number and density of network nodes) and local connectivity utilized for the analysis of the brain dynamics may affect the results (Proix et al., 2016). This issue plays a crucial role for the data-driven studies, where several parcellation schemes (atlases) are currently in use without a general consensus on their appropriateness (Zalesky et al., 2010; Eickhoff et al., 2018a). On the other hand, the level of the parcellation granularity, for example, may strongly impact the topological properties of brain networks already at the level of SC (Zalesky et al., 2010). At the level of functional interrelations between brain nodes, the directionality, weight and type of connections are important, which can be addressed by an intrinsically model-based approach of dynamic causal modeling (DCM) (Friston et al., 2003) providing an estimation of effective connectivity reflecting causal interactions. Albeit inferring the effective connectivity in large-scale whole-brain networks is a challenging problem, recent progress with DCM (Friston et al., 2014, 2017; Frassle et al., 2017) may support a prompt development in this field. 
Data analysis of the resting-state fMRI strongly relies on pre-processing steps, where confound regression is applied to clean fMRI recordings from contaminating signals (Murphy et al., 2013; Satterthwaite et al., 2013; Power et al., 2014; SalimiKhorshidi et al., 2014; Varikuti et al., 2017). The validation of the brain models also depends on the respective nuisance signal regression, and previous modeling studies showed that conclusion derived from the models may depend on the utilized data processing steps, in particular, on whether the global brain signal was regressed or not (Cabral et al., 2012b, 2013; Yang et al., 2014; Anticevic et al., 2015). For different confound regression and data processing approaches, the impact of those on the parameters of the validated models can be evaluated. In such a way the effects of the data pre-processing procedures can be parametrized, classified and compared to each other as well as to the results of the data-driven approach (Varikuti et al., 2017).

Dynamical models can be applied to the entire range of the subject cohorts ranging from personalized models optimized for a single individual to the models designed to investigate effects of ensemble averaging, where a few tens of subjects were usually considered in the latter case (Cabral et al., 2012a; Bettinardi et al., 2017). The explanatory and predictive power of the large-scale models may be enhanced if large subject cohorts get involved in the modeling analysis as it is for the data-driven approach (Satterthwaite et al., 2013; Finn et al., 2015; Liem et al., 2017). On the other hand, personalized dynamical brain models can be an important goal for the model-based approach because of their application potential for personalized diagnosis, prevention and treatment of diseases as exemplified by the concept of the virtual epileptic patient (Jirsa et al., 2017; Proix et al., 2017).

\section{REFERENCES}

Adamchic, I., Hauptmann, C., Barnikol, U. B., Pawelczyk, N., Popovych, O., Barnikol, T. T., et al. (2014). Coordinated reset neuromodulation for Parkinson's disease: proof-of-concept study. Mov. Disord. 29, 1679-1684. doi: $10.1002 / \mathrm{mds} .25923$

Allen, E. A., Damaraju, E., Plis, S. M., Erhardt, E. B., Eichele, T., and Calhoun, V. D. (2014). Tracking whole-brain connectivity dynamics in the resting state. Cereb. Cortex 24, 663-676. doi: 10.1093/cercor/bhs352

Anticevic, A., Hu, X. Y., Xiao, Y., Hu, J. M., Li, F., Bi, F., et al. (2015). Early-course unmedicated schizophrenia patients exhibit elevated prefrontal connectivity associated with longitudinal change. J. Neurosci. 35, 267-286. doi: 10.1523/JNEUROSCI.2310-14.2015

Arbabshirani, M. R., Plis, S., Sui, J., and Calhoun, V. D. (2017). Single subject prediction of brain disorders in neuroimaging: promises and pitfalls. Neuroimage 145, 137-165. doi: 10.1016/j.neuroimage.2016.02.079

Bandettini, P. A. (2009). What's new in neuroimaging methods? Ann. N.Y. Acad. Sci. 1156, 260-293. doi: 10.1111/j.1749-6632.2009.04420.x

Bettinardi, R. G., Deco, G., Karlaftis, V. M., Van Hartevelt, T. J., Fernandes, H. M., Kourtzi, Z., et al. (2017). How structure sculpts function: unveiling the contribution of anatomical connectivity to the brain's spontaneous correlation structure. Chaos 27:047409. doi: 10.1063/1.4980099

Biswal, B., Yetkin, F. Z., Haughton, V. M., and Hyde, J. S. (1995). Functional connectivity in the motor cortex of resting human brain using echo-planar MRI. Magn. Reson. Med. 34, 537-541. doi: 10.1002/mrm.1910340409

Bressler, S. L., and Menon, V. (2010). Large-scale brain networks in cognition: emerging methods and principles. Trends Cogn. Sci. 14, 277-290. doi: 10.1016/j.tics.2010.04.004
Other recent modeling studies suggest that model parameters may serve as potential biomarkers for disease diagnosis (Cabral et al., 2012b, 2013; van Hartevelt et al., 2014; Falcon et al., 2016). Such personalized models can also be used for the development and computational testing of personalized treatment of diseases.

In summary, there are several promising applications of the model-based approach, which could advance neuroimaging data analytics and translational medicine. To achieve this, an interdisciplinary research on data analysis should also include expertise from nonlinear dynamical systems and mathematics, focus on detailed investigation of the models and compare their results with the data.

\section{AUTHOR CONTRIBUTIONS}

OP, TM, FH, and SE contributed to the conception of the work. OP and TM drafted the manuscript. All the authors revised the manuscript and approved the final version of the manuscript.

\section{ACKNOWLEDGMENTS}

The authors gratefully acknowledge helpful discussions with Viktor Jirsa and Gustavo Deco. This work was supported by the Deutsche Forschungsgemeinschaft (DFG, EI 816/11-1), the National Institute of Mental Health (R01-MH074457), the Helmholtz Portfolio Theme Supercomputing and Modeling for the Human Brain and the European Union's Horizon 2020 Research and Innovation Programme under Grant Agreement 720270 (HBP SGA1) and 785907 (HBP SGA2).

Bullmore, E. T., and Bassett, D. S. (2011). Brain graphs: graphical models of the human brain connectome. Annu. Rev. Clin. Psych. 7, 113-140. doi: 10.1146/annurev-clinpsy-040510-143934

Cabral, J., Fernandes, H. M., Van Hartevelt, T. J., James, A. C., Kringelbach, M. L., and Deco, G. (2013). Structural connectivity in schizophrenia and its impact on the dynamics of spontaneous functional networks. Chaos 23:046111

Cabral, J., Hugues, E., Kringelbach, M. L., and Deco, G. (2012a). Modeling the outcome of structural disconnection on resting-state functional connectivity. Neuroimage 62, 1342-1353. doi: 10.1016/j.neuroimage.2012.06.007

Cabral, J., Hugues, E., Sporns, O., and Deco, G. (2011). Role of local network oscillations in resting-state functional connectivity. Neuroimage 57, 130-139. doi: 10.1016/j.neuroimage.2011.04.010

Cabral, J., Kringelbach, M. L., and Deco, G. (2012b). Functional graph alterations in schizophrenia: a result from a global anatomic decoupling? Pharmacopsychiatry 45, S57-S64. doi: 10.1055/s-0032-1309001

Cabral, J., Kringelbach, M. L., and Deco, G. (2017a). Functional connectivity dynamically evolves on multiple time-scales over a static structural connectome: Models and mechanisms. Neuroimage 160, 84-96. doi: 10.1016/j.neuroimage.2017.03.045

Cabral, J., Vidaurre, D., Marques, P., Magalhães, R., Silva Moreira, P., Miguel Soares, J., et al. (2017b). Cognitive performance in healthy older adults relates to spontaneous switching between states of functional connectivity during rest. Sci. Rep. 7:5135

Calhoun, V. D., Eichele, T., and Pearlson, G. (2009). Functional brain networks in schizophrenia: a review. Front. Hum. Neurosci. 3:17. doi: 10.3389/neuro.09.017.2009

Carnevale, N. T., and Hines, M. L. (2006). The NEURON Book. Cambridge, UK: Cambridge University Press. doi: 10.1017/CBO9780511541612 
Caspers, S., Moebus, S., Lux, S., Pundt, N., Schutz, H., Muhleisen, T. W., et al. (2014). Studying variability in human brain aging in a population-based german cohort-rationale and design of 1000 BRAINS. Front. Aging Neurosci. 6:149. doi: 10.3389/fnagi.2014.00149

Cocchi, L., Gollo, L. L., Zalesky, A., and Breakspear, M. (2017). Criticality in the brain: a synthesis of neurobiology, models and cognition. Prog. Neurobiol. 158, 132-152. doi: 10.1016/j.pneurobio.2017.07.002

Damaraju, E., Allen, E. A., Belger, A., Ford, J. M., McEwen, S., Mathalon, D. H., et al. (2014). Dynamic functional connectivity analysis reveals transient states of dysconnectivity in schizophrenia. Neuroimage Clin. 5, 298-308. doi: 10.1016/j.nicl.2014.07.003

Deco, G., Jirsa, V., McIntosh, A. R., Sporns, O., and Kötter, R. (2009). Key role of coupling, delay, and noise in resting brain fluctuations. Proc. Natl. Acad. Sci. U.S.A. 106, 10302-10307. doi: 10.1073/pnas.0901831106

Deco, G., and Jirsa, V. K. (2012). Ongoing cortical activity at rest: Criticality, multistability, and ghost attractors. J. Neurosci. 32, 3366-3375. doi: 10.1523/JNEUROSCI.2523-11.2012

Deco, G., Jirsa, V. K., and McIntosh, A. R. (2013). Resting brains never rest: computational insights into potential cognitive architectures. Trends Neurosci. 36, 268-274. doi: 10.1016/j.tins.2013.03.001

Deco, G., Jirsa, V. K., Robinson, P. A., Breakspear, M., and Friston, K. (2008). The dynamic brain: from spiking neurons to neural masses and cortical fields. PLoS Comput. Biol. 4:e1000092. doi: 10.1371/journal.pcbi. 1000092

Deco, G., and Kringelbach, M. L. (2014). Great expectations: using whole-brain computational connectomics for understanding neuropsychiatric disorders. Neuron 84, 892-905. doi: 10.1016/j.neuron.2014. 08.034

Deco, G., and Kringelbach, M. L. (2016). Metastability and coherence: extending the communication through coherence hypothesis using a whole-brain computational perspective. Trends Neurosci. 39, 125-135. doi: 10.1016/j.tins.2016.01.001

Deco, G., Kringelbach, M. L., Jirsa, V. K., and Ritter, P. (2017). The dynamics of resting fluctuations in the brain: metastability and its dynamical cortical core. Sci. Rep. 7:3095. doi: 10.1038/s41598-017-03073-5

Deco, G., McIntosh, A. R., Shen, K., Hutchison, R. M., Menon, R. S., Everling, S., et al. (2014a). Identification of optimal structural connectivity using functional connectivity and neural modeling. J. Neurosci. 34, 7910-7916. doi: 10.1523/JNEUROSCI.4423-13.2014

Deco, G., Ponce-Alvarez, A., Hagmann, P., Romani, G. L., Mantini, D., and Corbetta, M. (2014b). How local excitation-inhibition ratio impacts the whole brain dynamics. J. Neurosci. 34, 7886-7898. doi: 10.1523/JNEUROSCI.5068-13.2014

Deco, G., Senden, M., and Jirsa, V. (2012). How anatomy shapes dynamics: a semianalytical study of the brain at rest by a simple spin model. Front. Comput. Neurosci. 6:68. doi: 10.3389/fncom.2012.00068

Dosenbach, N. U., Nardos, B., Cohen, A. L., Fair, D. A., Power, J. D., Church, J. A., et al. (2010). Prediction of individual brain maturity using fMRI. Science 329, 1358-1361. doi: 10.1126/science.1194144

Dubois, J., and Adolphs, R. (2016). Building a science of individual differences from fMRI. Trends Cogn. Sci. 20, 425-443. doi: 10.1016/j.tics.2016.03.014

Duffy, D. J. (2016). Problems, challenges and promises: perspectives on precision medicine. Briefings Bioinf. 17, 494-504. doi: 10.1093/bib/bbv060

Ebert, M., Hauptmann, C., and Tass, P. (2014). Coordinated reset stimulation in a large-scale model of the STN-GPe circuit. Front. Comput. Neurosci. 8:154. doi: $10.3389 /$ fncom.2014.00154

Eickhoff, S. B., Constable, R. T., and Yeo, B. T. T. (2018a). Topographic organization of the cerebral cortex and brain cartography. Neuroimage 170, 332-347. doi: 10.1016/j.neuroimage.2017.02.018

Eickhoff, S. B., Yeo, B. T. T., and Genon, S. (2018b). Imaging-based parcellations of the human brain. Nat. Rev. Neurosci. 19, 672-686. doi: 10.1038/s41583-018-0071-7

Falcon, M. I., Riley, J. D., Jirsa, V., McIntosh, A. R., Chen, E. E., and Solodkin, A. (2016). Functional mechanisms of recovery after chronic stroke: Modeling with The Virtual Brain. Eneuro 3:UNSP e0158-15.2016. doi: 10.1523/ENEURO.0158-15.2016

Finn, E. S., Shen, X. L., Scheinost, D., Rosenberg, M. D., Huang, J., Chun, M. M., et al. (2015). Functional connectome fingerprinting: identifying individuals using patterns of brain connectivity. Nat. Neurosci. 18, 1664-1671. doi: 10.1038/nn.4135

Fox, M. D., Buckner, R. L., Liu, H. S., Chakravarty, M. M., Lozano, A. M., and Pascual-Leone, A. (2014). Resting-state networks link invasive and noninvasive brain stimulation across diverse psychiatric and neurological diseases. Proc. Natl. Acad. Sci. U.S.A. 111, E4367-E4375. doi: 10.1073/pnas.1405003111

Fox, M. D., Zhang, D. Y., Snyder, A. Z., and Raichle, M. E. (2009). The global signal and observed anticorrelated resting state brain networks. J. Neurophysiol. 101, 3270-3283. doi: 10.1152/jn.90777.2008

Frässle, S., Lomakina, E. I., Razi, A., Friston, K. J., Buhmann, J. M., and Stephan, K. E. (2017). Regression DCM for fMRI. Neuroimage 155, 406-421. doi: 10.1016/j.neuroimage.2017.02.090

Friston, K. J., Preller, K. H., Mathys, C., Cagnan, H., Heinzle, J., Razi, A., et al. (2017). Dynamic causal modelling revisited. Neuroimage doi: 10.1016/j.neuroimage.2017.02.045. [Epub ahead of print].

Friston, K. J., and Frith, C. D. (1995). Schizophrenia - a disconnection syndrome. Clin. Neurosci. 3, 89-97.

Friston, K. J., Harrison, L., and Penny, W. (2003). Dynamic causal modelling. Neuroimage 19, 1273-1302. doi: 10.1016/S1053-8119(03)00202-7

Friston, K. J., Kahan, J., Biswal, B., and Razi, A. (2014). A DCM for resting state fMRI. Neuroimage 94, 396-407. doi: 10.1016/j.neuroimage.2013.12.009

Gewaltig, M., and Diesmann, M. (2007). NEST (NEural Simulation Tool). Scholarpedia 2:1430. doi: 10.4249/scholarpedia.1430

Ghosh, A., Rho, Y., McIntosh, A. R., Kötter, R., and Jirsa, V. K. (2008). Noise during rest enables the exploration of the brain's dynamic repertoire. PLoS Comput. Biol. 4:e1000196. doi: 10.1371/journal.pcbi.1000196

Glomb, K., Ponce-Alvarez, A., Gilson, M., Ritter, P., and Deco, G. (2017). Resting state networks in empirical and simulated dynamic functional connectivity. Neuroimage 159, 388-402. doi: 10.1016/j.neuroimage.2017.07.065

Golos, M., Jirsa, V., and Daucé, E. (2015). Multistability in large scale models of brain activity. PLoS Comput. Biol. 11:e1004644. doi: 10.1371/journal.pcbi.1004644

Goodman, D. F., and Brette, R. (2009). The brian simulator. Front. Neurosci. 3, 192-197. doi: 10.3389/neuro.01.026.2009

Hagmann, P., Cammoun, L., Gigandet, X., Meuli, R., Honey, C. J., Wedeen, V., et al. (2008). Mapping the structural core of human cerebral cortex. PLoS Biol. 6:e159. doi: 10.1371/journal.pbio.0060159

Hansen, E. C. A., Battaglia, D., Spiegler, A., Deco, G., and Jirsa, V. K. (2015). Functional connectivity dynamics: Modeling the switching behavior of the resting state. Neuroimage 105, 525-535. doi: 10.1016/j.neuroimage.2014.11.001

Hermundstad, A. M., Bassett, D. S., Brown, K. S., Aminoff, E. M., Clewett, D., Freeman, S., et al. (2013). Structural foundations of resting-state and task-based functional connectivity in the human brain. Proc. Natl. Acad. Sci. U.S.A. 110, 6169-6174. doi: 10.1073/pnas.1219562110

Honey, C. J., Sporns, O., Cammoun, L., Gigandet, X., Thiran, J. P., Meuli, R., et al. (2009). Predicting human resting-state functional connectivity from structural connectivity. Proc. Natl. Acad. Sci. U.S.A. 106, 2035-2040. doi: 10.1073/pnas.0811168106

Horwitz, B., Friston, K. J., and Taylor, J. G. (2000). Neural modeling and functional brain imaging: an overview. Neur. Netw. 13, 829-846 doi: 10.1016/S0893-6080(00)00062-9

Hutchison, R. M., Womelsdorf, T., Allen, E. A., Bandettini, P. A., Calhoun, V. D., Corbetta, M., et al. (2013). Dynamic functional connectivity: Promise, issues, and interpretations. Neuroimage 80, 360-378. doi: 10.1016/j.neuroimage.2013.05.079

Jirsa, V. K., Proix, T., Perdikis, D., Woodman, M. M., Wang, H., GonzalezMartinez, J., et al. (2017). The virtual pileptic Patient: individualized whole-brain models of epilepsy spread. Neuroimage 145, 377-388. doi: 10.1016/j.neuroimage.2016.04.049

Jobst, B. M., Hindriks, R., Laufs, H., Tagliazucchi, E., Hahn, G., Ponce-Alvarez, A., et al. (2017). Increased stability and breakdown of brain effective connectivity during slow-wave sleep: mechanistic insights from whole-brain computational modelling. Sci. Rep. 7:4634. doi: 10.1038/s41598-017-04522-x

Jones, D. K., Knösche, T. R., and Turner, R. (2013). White matter integrity, fiber count, and other fallacies: the do's and don'ts of diffusion MRI. Neuroimage 73 239-254. doi: 10.1016/j.neuroimage.2012.06.081

Knock, S. A., McIntosh, A. R., Sporns, O., Kotter, R., Hagmann, P., and Jirsa, V. K. (2009). The effects of physiologically plausible connectivity structure on local 
and global dynamics in large scale brain models. J. Neurosci. Methods 183, 86-94. doi: 10.1016/j.jneumeth.2009.07.007

Konrad, K., and Eickhoff, S. B. (2010). Is the ADHD brain wired differently? a review on structural and functional connectivity in attention deficit hyperactivity disorder. Hum. Brain Mapp. 31, 904-916. doi: 10.1002/hbm.21058

Liem, F., Varoquaux, G., Kynast, J., Beyer, F., Masouleh, S. K., Huntenburg, J. M., et al. (2017). Predicting brain-age from multimodal imaging data captures cognitive impairment. Neuroimage 148, 179-188. doi: 10.1016/j.neuroimage.2016.11.005

Little, S., Pogosyan, A., Neal, S., Zavala, B., Zrinzo, L., Hariz, M., et al. (2013). Adaptive deep brain stimulation in advanced Parkinson disease. Ann. Neurol. 74, 449-457. doi: 10.1002/ana.23951

Lynall, M. E., Bassett, D. S., Kerwin, R., McKenna, P. J., Kitzbichler, M., Muller, U., et al. (2010). Functional connectivity and brain networks in schizophrenia. J. Neurosci. 30, 9477-9487. doi: 10.1523/JNEUROSCI.0333-10.2010

Maier-Hein, K. H., Neher, P. F., Houde, J. C., Côté, M.-A., Garyfallidis, E., Zhong, J., et al. (2017). The challenge of mapping the human connectome based on diffusion tractography. Nat. Commun. 8:1349

Manos, T., Zeitler, M., and Tass, P. A. (2018a). How stimulation frequency and intensity impact on the long-lasting effects of coordinated reset stimulation. PLoS Comput. Biol. 14, 1-31. doi: 10.1371/journal.pcbi.1006113

Manos, T., Zeitler, M., and Tass, P. A. (2018b). Short-term dosage regimen for stimulation-induced long-lasting desynchronization. Front. Physiol. 9:376. doi: 10.3389/fphys.2018.00376

Marrelec, G., Messé, A., Giron, A., and Rudrauf, D. (2016). Functional connectivity's degenerate view of brain computation. PLoS Comput. Biol. 12:e1005031. doi: 10.1371/journal.pcbi.1005031

Murphy, K., Birn, R. M., and Bandettini, P. A. (2013). Restingstate fMRI confounds and cleanup. Neuroimage 80, 349-359. doi: 10.1016/j.neuroimage.2013.04.001

Murphy, K., Birn, R. M., Handwerker, D. A., Jones, T. B., and Bandettini, P. A. (2009). The impact of global signal regression on resting state correlations: Are anti-correlated networks introduced? Neuroimage 44, 893905. doi: 10.1016/j.neuroimage.2008.09.036

Naik, S., Banerjee, A., Bapi, R. S., Deco, G., and Roy, D. (2017). Metastability in senescence. Trends Cogn. Sci. 21, 509-521. doi: 10.1016/j.tics.2017.04.007

Nakagawa, T. T., Jirsa, V. K., Spiegler, A., McIntosh, A. R., and Deco, G. (2013). Bottom up modeling of the connectome: Linking structure and function in the resting brain and their changes in aging. Neuroimage 80, 318-329. doi: 10.1016/j.neuroimage.2013.04.055

Park, H. J., and Friston, K. J. (2013). Structural and functional brain networks: From connections to cognition. Science 342, 1238411-1238418. doi: $10.1126 /$ science.1238411

Pläschke, R. N., Cieslik, E. C., Müller, V. I., Hoffstaedter, F., Plachti, A., Varikuti, D. P., et al. (2017). On the integrity of functional brain networks in schizophrenia, Parkinson's disease, and advanced age: Evidence from connectivity-based single-subject classification. Hum. Brain Mapp. 38, 58455858. doi: 10.1002/hbm. 23763

Ponce-Alvarez, A., Deco, G., Hagmann, P., Romani, G. L., Mantini, D., and Corbetta, M. (2015). Resting-state temporal synchronization networks emerge from connectivity topology and heterogeneity. PLoS Comput. Biol. 11:UNSP e1004100. doi: 10.1371/journal.pcbi.1004100

Popovych, O. V., Hauptmann, C., and Tass, P. A. (2005). Effective desynchronization by nonlinear delayed feedback. Phys. Rev. Lett. 94:164102

Popovych, O. V., Hauptmann, C., and Tass, P. A. (2006). Control of neuronal synchrony by nonlinear delayed feedback. Biol. Cybern. 95, 69-85. doi: 10.1007/s00422-006-0066-8

Popovych, O. V., Lysyansky, B., Rosenblum, M., Pikovsky, A., and Tass, P. A. (2017a). Pulsatile desynchronizing delayed feedback for closed-loop deep brain stimulation. PLoS ONE 12:e0173363. doi: 10.1371/journal.pone.0173363

Popovych, O. V., Lysyansky, B., and Tass, P. A. (2017b). Closed-loop deep brain stimulation by pulsatile delayed feedback with increased gap between pulse phases. Sci. Rep. 7:1033. doi: 10.1038/s41598-01701067-x

Popovych, O. V., and Tass, P. A. (2012). Desynchronizing electrical and sensory coordinated reset neuromodulation. Front. Hum. Neurosci. 6:58. doi: $10.3389 /$ fnhum. 2012.00058
Popovych, O. V., and Tass, P. A. (2018). Multisite delayed feedback for electrical brain stimulation. Front. Physiol. 9:46. doi: 10.3389/fphys.2018.00046

Popovych, O. V., Yanchuk, S., and Tass, P. A. (2013). Self-organized noise resistance of oscillatory neural networks with spike timing-dependent plasticity. Sci. Rep. 3:2926 doi: 10.1038/srep02926

Power, J. D., Mitra, A., Laumann, T. O., Snyder, A. Z., Schlaggar, B. L., and Petersen, S. E. (2014). Methods to detect, characterize, and remove motion artifact in resting state fMRI. Neuroimage 84, 320-341. doi: 10.1016/j.neuroimage.2013.08.048

Preti, M. G., Bolton, T. A. W., and Van De Ville, D. (2017). The dynamic functional connectome: state-of-the-art and perspectives. Neuroimage 160, 41-54. doi: 10.1016/j.neuroimage.2016.12.061

Proix, T., Bartolomei, F., Guye, M., and Jirsa, V. K. (2017). Individual brain structure and modelling predict seizure propagation. Brain 140, 641-654. doi: 10.1093/brain/awx004

Proix, T., Spiegler, A., Schirner, M., Rothmeier, S., Ritter, P., and Jirsa, V. K. (2016). How do parcellation size and short-range connectivity affect dynamics in large-scale brain network models? Neuroimage 142, 135-149. doi: 10.1016/j.neuroimage.2016.06.016

Rabinovich, M. I., Huerta, R., Varona, P., and Afraimovich, V. S. (2008). Transient cognitive dynamics, metastability, and decision making. PLoS Comput. Biol. 4:e1000072. doi: 10.1371/journal.pcbi.1000072

Rashid, B., Damaraju, E., Pearlson, G. D., and Calhoun, V. D. (2014). Dynamic connectivity states estimated from resting fmri identify differences among schizophrenia, bipolar disorder, and healthy control subjects. Front. Hum. Neurosci. 8:897. doi: 10.3389/fnhum.2014.00897

Ritter, P., Schirner, M., McIntosh, A. R., and Jirsa, V. K. (2013). The Virtual Brain integrates computational modeling and multimodal neuroimaging. Brain Connect. 3, 121-145. doi: 10.1089/brain.2012.0120

Rosa, M., Arlotti, M., Ardolino, G., Cogiamanian, F., Marceglia, S., Di Fonzo, A., et al. (2015). Adaptive deep brain stimulation in a freely moving parkinsonian patient. Mov. Disord. 30, 1003-1005. doi: 10.1002/mds.26241

Rosenblum, M. G., and Pikovsky, A. S. (2004). Delayed feedback control of collective synchrony: An approach to suppression of pathological brain rhythms. Phys. Rev. E 70:041904. doi: 10.1103/PhysRevE.70.041904

Saenger, V. M., Kahan, J., Foltynie, T., Friston, K., Aziz, T. Z., Green, A. L., et al. (2017). Uncovering the underlying mechanisms and whole-brain dynamics of deep brain stimulation for Parkinson's disease. Sci. Reports 7:9882. doi: 10.1038/s41598-017-10003-y

Salimi-Khorshidi, G., Douaud, G., Beckmann, C. F., Glasser, M. F., Griffanti, L., and Smith, S. M. (2014). Automatic denoising of functional MRI data: combining independent component analysis and hierarchical fusion of classifiers. Neuroimage 90, 449-468. doi: 10.1016/j.neuroimage.2013.11.046

Sanz Leon, P., Knock, S. A., Woodman, M. M., Domide, L., Mersmann, J., McIntosh, A. R., et al. (2013). The Virtual Brain: a simulator of primate brain network dynamics. Front. Neuroinf. 7:10. doi: 10.3389/fninf.2013.00010

Sanz-Leon, P., Knock, S. A., Spiegler, A., and Jirsa, V. K. (2015). Mathematical framework for large-scale brain network modeling in The Virtual Brain. Neuroimage 111, 385-430. doi: 10.1016/j.neuroimage.2015.01.002

Satterthwaite, T. D., Elliott, M. A., Gerraty, R. T., Ruparel, K., Loughead, J., Calkins, M. E., et al. (2013). An improved framework for confound regression and filtering for control of motion artifact in the preprocessing of resting-state functional connectivity data. Neuroimage 64, 240-256. doi: 10.1016/j.neuroimage.2012.08.052

Schirner, M., McIntosh, A. R., Jirsa, V., Deco, G., and Ritter, P. (2018). Inferring multi-scale neural mechanisms with brain network modelling. eLife 7:e28927. doi: 10.7554/eLife.28927

Shen, X. L., Finn, E. S., Scheinost, D., Rosenberg, M. D., Chun, M. M., Papademetris, X., et al. (2017). Using connectome-based predictive modeling to predict individual behavior from brain connectivity. Nature Protocols 12, 506-518. doi: 10.1038/nprot.2016.178

Sorg, C., Riedl, V., Muhlau, M., Calhoun, V. D., Eichele, T., Läer, L., et al. (2007). Selective changes of resting-state networks in individuals at risk for Alzheimer's disease. Proc. Natl. Acad. Sci. U.S.A. 104, 18760-18765. doi: 10.1073/pnas.0708803104

Sporns, O., Tononi, G., and Kotter, R. (2005). The human connectome: a structural description of the human brain. PLoS Comput. Biol. 1:e42. doi: 10.1371/journal.pcbi.0010042 
Stephan, K. E., Iglesias, S., Heinzle, J., and Diaconescu, A. O. (2015). Translational perspectives for computational neuroimaging. Neuron 87, 716732. doi: 10.1016/j.neuron.2015.07.008

Tahmasian, M., Bettray, L. M., van Eimeren, T., Drzezga, A., Timmermann, L., Eickhoff, C. R., et al. (2015). A systematic review on the applications of restingstate fMRI in Parkinson's disease: Does dopamine replacement therapy play a role? Cortex 73, 80-105. doi: 10.1016/j.cortex.2015.08.005

Tass, P. A. (2003). A model of desynchronizing deep brain stimulation with a demand-controlled coordinated reset of neural subpopulations. Biol. Cybern. 89, 81-88. doi: 10.1007/s00422-003-0425-7

Tass, P. A., Adamchic, I., Freund, H.-J., von Stackelberg, T., and Hauptmann, C. (2012a). Counteracting tinnitus by acoustic coordinated reset neuromodulation. Rest. Neurol. Neurosci. 30, 137-159. doi: 10.3233/RNN-2012-110218

Tass, P. A., and Majtanik, M. (2006). Long-term anti-kindling effects of desynchronizing brain stimulation: a theoretical study. Biol. Cybern. 94, 58-66. doi: 10.1007/s00422-005-0028-6

Tass, P. A., Qin, L., Hauptmann, C., Doveros, S., Bezard, E., Boraud, T., et al. (2012b). Coordinated reset has sustained aftereffects in parkinsonian monkeys. Ann. Neurol. 72, 816-820. doi: 10.1002/ana.23663

Tavor, I., Jones, O. P., Mars, R. B., Smith, S. M., Behrens, T. E., and Jbabdi, S. (2016). Task-free MRI predicts individual differences in brain activity during task performance. Science 352, 216-220. doi: 10.1126/science.aad8127

Thirion, B., Varoquaux, G., Dohmatob, E., and Poline, J.-B. (2014). Which fMRI clustering gives good brain parcellations? Front. Neurosci. 8:167. doi: 10.3389/fnins.2014.00167

Thomas, C., Ye, F. Q., Irfanoglu, M. O., Modi, P., Saleem, K. S., Leopold, D. A., et al. (2014). Anatomical accuracy of brain connections derived from diffusion MRI tractography is inherently limited. Proc. Natl. Acad. Sci. U.S.A. 111, 16574-16579. doi: 10.1073/pnas.1405672111

Tognoli, E., and Kelso, J. A. S. (2014). The metastable brain. Neuron 81, 35-48. doi: 10.1016/j.neuron.2013.12.022

Ton, R., Deco, G., and Daffertshofer, A. (2014). Structure-function discrepancy: inhomogeneity and delays in synchronized neural networks. PLoS Comput. Biol. 10:e1003736. doi: 10.1371/journal.pcbi.1003736

van den Heuvel, M. P. and Fornito, A. (2014). Brain networks in schizophrenia. Neuropsychol. Rev. 24, 32-48. doi: 10.1007/s11065-014-9248-7
Van Essen, D. C., Smith, S. M., Barch, D. M., Behrens, T. E. J., Yacoub, E., and Ugurbil, K. (2013). The WU-Minn Human Connectome Project: an overview. Neuroimage 80, 62-79. doi: 10.1016/j.neuroimage.2013.05.041

van Hartevelt, T. J., Cabral, J., Deco, G., Moller, A., Green, A. L., Aziz, T. Z., et al. (2014). Neural plasticity in human brain connectivity: the effects of long term deep brain stimulation of the subthalamic nucleus in parkinson's disease. PLoS ONE 9:e86496. doi: 10.1371/journal.pone.0086496

Varikuti, D. P., Hoffstaedter, F., Genon, S., Schwender, H., Reid, A. T., and Eickhoff, S. B. (2017). Resting-state test-retest reliability of a priori defined canonical networks over different preprocessing steps. Brain Struct. Funct. 222, 1447-1468. doi: 10.1007/s00429-016-1286-x

Woo, C. W., Chang, L. J., Lindquist, M. A., and Wager, T. D. (2017). Building better biomarkers: brain models in translational neuroimaging. Nat. Neurosci. 20, 365-377. doi: 10.1038/nn.4478

Yahata, N., Kasai, K., and Kawato, M. (2017). Computational neuroscience approach to biomarkers and treatments for mental disorders. Psychiatry Clin. Neurosci. 71, 215-237. doi: 10.1111/pcn.12502

Yang, G. J., Murray, J. D., Repovs, G., Cole, M. W., Savic, A., Glasser, M. F., et al. (2014). Altered global brain signal in schizophrenia Proc. Natl. Acad. Sci. U.S.A. 111, 7438-7443. doi: 10.1073/pnas. 1405289111

Zalesky, A., Fornito, A., Harding, I. H., Cocchi, L., Yucel, M., Pantelis, C., et al. (2010). Whole-brain anatomical networks: Does the choice of nodes matter? Neuroimage 50, 970-983. doi: 10.1016/j.neuroimage.2009. 12.027

Conflict of Interest Statement: The authors declare that the research was conducted in the absence of any commercial or financial relationships that could be construed as a potential conflict of interest.

Copyright (c) 2019 Popovych, Manos, Hoffstaedter and Eickhoff. This is an openaccess article distributed under the terms of the Creative Commons Attribution License (CC BY). The use, distribution or reproduction in other forums is permitted, provided the original author(s) and the copyright owner(s) are credited and that the original publication in this journal is cited, in accordance with accepted academic practice. No use, distribution or reproduction is permitted which does not comply with these terms. 